\title{
DISEÑO DE UN HORNO PILOTO PARA FABRICACIÓN DE HIERRO ESPONJA
}

\author{
Zoilo Valdivia Infantas ${ }^{\prime}$; Julián Nieto Quispe ${ }^{I}$; \\ Daniel Zevallos Ramos ${ }^{1}$; Matias Vivar Colquicocha ${ }^{1}$
}

\author{
RESUMEN
}

El presente trabajo realizado del 01 de enero al 08 de setiembre del 2000, se refiere al diseño y construcción de un Horno Piloto para la fabricación de Hierro Esponja, que permita obtenerlo directamente del mineral y a un costo por debajo del precio de la chatarra y que pueda servir como precipitador de cobre a partir de soluciones de sulfato de cobre.

\section{A B S TR A C T}

this research work was done from january 01 to september 08 in 2000. it refers to he design and building of a pilot oven for the manufacture of sponge iron which allows to obtain it directly from the mineral and at a very low prize, even a prize below the scrap prize, which can also be used as a copper precipitator obtained from copper sulfate solutions.

\section{OBJETIVO}

El principal objetivo es promover la explotación de los yacimientos de oxido de cobre que existen en la zona de Tacna, empleando el mineral de hierro y el carbón existentes en la zona, para lo cual es necesario demostrar que el hierro esponja puede servir como precipitador del cobre de sus soluciones de sulfato, que se puede poducir un hierro esponja más barato que la chatarra o, en todo caso, superar el problema de su disponibilidad en la zona, y que el estudio de factibilidad de explotación de un yacimiento específico, tanto de cobre como de hierro y carbón, indique una rentabilidad aceptable.

\section{UBICACIÓN}

El horno se está construyendo en el Laboratorio de Transformación y Tratamientos Térmicos de la Facultad de Ingenieria Metalúrgica (FAME) de la Universidad Nacional Jorge Basadre Grohmann (UNJBG), y las pruebas que se derivarán y que serán motivo de otro trabajo de investigación se realizarán también en dicho Laboratorio y en el Laboratorio de
Hidrometalurgia de la FAME.

\section{METODOLOGIA}

En el desarrollo del presente trabajo se han seguido los siguientes pasos:

- Se efectuó la acumulación de información, tanto teórica como de experiencia, sobre todo de la tecnología existente del país.

- De las diferentes alternativas técnicas, se optó por una combinación de los principios básicos de los procesos SL/RN y MIDREX, introduciendo las modificaciones necesarias.

- Se efectuaron los cálculos para determinar los parámetros de diseño para diferentes tamaños del horno, sobre todo teniendo en cuenta el peso de carga y el costo de las prácticas académicas que se llevarán a cabo con este equipo, así como la seguridad en su operación.

1 Ingeniero Metalúrgico 
- Se efectuó una simulación teórica de funcionamiento del equipo, obteniéndose los balances de materia y de energía.

\section{INTRODUCCIÓN}

El objetivo del presente trabajo es el diseño y la construcción de un Horno Piloto de reducción directa de minerales de hierro, que sirva para realizar pruebas de bajo costo para sustentar estudios técnicos para la explotación de los yacimientos de minerales de hierro que existen en la Zona Sur del pais con la finalidad de producir Hierro Esponja, de determinadas características, con la posibilidad de ser usado en la producción de cemento de cobre, generándose así una buena alternativa para la explotación de los yacimientos de óxidos de cobre, bastante abundantes en las provincias de Tacna, al poder reemplazar la chatarra, a veces de difícil disponibilidad, con el Hierro Esponja.

El proceso de reducción directa de los minerales de hierro permite producir el Hierro Esponja, que es un producto generalmente en forma de esferas porosas de $1 \mathrm{~cm}$ de diámetro, cuando el mineral de hierro ha sido previamente triturado, concentrado y peletizado. Los poros representan aproximadamente el $30 \%$ del volumen de la esfera.

Uno de los objetivos de las pruebas que se van a realizar en este Horno Piloto es el de producir Hierro
Esponja directamente del mineral a una granulometría de menos $1 / 4$ de pulgada.

El Hierro Esponja tiene un grado de metalización de 85 a $93 \%$, es decir, está constituido fundamentalmente de hierro metálico como resultado de la reducción del óxido de hierro por acción del monóxido de carbono a temperaturas del orden de los $1000^{\circ} \mathrm{C}$.

El principal uso del Hierro Esponja es el de servir como parte de la carga de los hornos eléctricos para producir el acero en plantas de menor capacidad.

En la Zona Sur del país y en Tacna, tenemos yacimientos de mineral de hierro que pueden ser explotados, siempre que se encuentre una alternativa técnica, económica y de mediana escala.

Una alternativa es la de sustituir la chatarra con el Hierro Esponja en los procesos hidrometalúrgicos de producción de cemento de cobre a partir de soluciones de sulfato de cobre en los que actúa como precipitador del cobre, por su poder reductor respecto al cobre, técnica que puede servir para desarrollar una Tecnología propia de bajo costo y de aplicación en la zonas donde existan yacimientos de óxido de cobre, lo cual requiere la construcción de un equipo piloto que permita realizar la investigación y experimentación necesaria.

\section{FISICO - QUÍMICA DEL PROCESO}

\subsection{REDUCCIÓN CON CO}

\section{REACCIÓN}

\section{CALOR DE REACCIÓN}


A $1000^{\circ} \mathrm{C}$ se realiza la reducción directa

$$
\begin{aligned}
& \mathrm{FeO}+\mathrm{CO}=\mathrm{Fe}+\mathrm{CO}_{2} \quad-3,2 \mathrm{Kal} / \mathrm{mol} \mathrm{g} \quad \mathrm{CO} \text { (Exot) } \\
& \mathrm{CO}_{2}+\mathrm{C}=2 \mathrm{CO}
\end{aligned}+41,2 \mathrm{Kcal} / \mathrm{mol} \mathrm{g} \quad \mathrm{CO} \text { (Endot) }
$$

$\mathrm{FeO}+\mathrm{C}=\mathrm{Fe}+\mathrm{CO} \mathrm{AH}=+38,0 \mathrm{Kcal} / \mathrm{mol} . \mathrm{g} \quad \mathrm{CO}$ (Endot)

\subsection{REDUCCIÓN CON $\mathrm{H}_{2}$}

$$
\begin{array}{lc}
\text { REACCIÓN } & \text { CALOR DE REACCIÓN } \\
3 \mathrm{Fe} 2 \mathrm{O} 3+\mathrm{H} 2=2 \mathrm{Fe} 3 \mathrm{O} 4+\mathrm{H} 2 \mathrm{O} & -8263 \mathrm{Kcal} / \mathrm{tFe}(\text { Exot }) \\
\mathrm{Fe} 3 \mathrm{O} 4+\mathrm{H} 2=3 \mathrm{FeO}+\mathrm{H} 2 \mathrm{O} & +110600 \mathrm{Kcal} / \mathrm{tFe}(\text { Endo }) \\
\mathrm{FeO}+\mathrm{H} 2=\mathrm{Fe}+\mathrm{H} 2 \mathrm{O} & +102614 \mathrm{Kcal} / \mathrm{Fe}(\text { Endo }) \\
\hline \mathrm{Fe} 2 \mathrm{O} 3+3 \mathrm{H} 2=2 \mathrm{Fe}+3 \mathrm{H} 2 \mathrm{O} & +204951 \mathrm{Kcal} / \mathrm{Fe}(\text { Endo })
\end{array}
$$

\section{MECANISMO DE LA REDUCCIÓN DIRECTA}

6.1. Difusión del co a través del lecho de partículas, el cual deberá tener la permeabilidad necesaria, controlada por la distribución granulométrica del mineral.

6.2. Difusión del CO a través de la capa límite que se forma alrededor de las partículas del mineral bajo un flujo de gas de $\mathrm{CO}$.

La capa límite ésta constituida por todas las moléculas de CO cuyo movimiento es retardado por fuerzas de fricción que se desarrollan por el contacto con las superficies de las partículas.

6.3. Dificultad del CO a través de la masa porosa que se produce por el oxígeno removido.

\begin{tabular}{|c|c|c|c|}
\hline \multicolumn{2}{|c|}{ ÓX1DO } & $\% \mathrm{Fe}$ & $\% \mathrm{O}_{2}$ \\
\hline WUSTITA & $\mathrm{Fe} O$ & 78 & 22 \\
\hline MAGNETITA & $\mathrm{Fe}_{3} \mathrm{O}_{4}$ & 73 & 27 \\
\hline HEMATITA & $\mathrm{Fe}_{2} \mathrm{O}_{3}$ & 70 & 30 \\
\hline
\end{tabular}

6.4. Difusión de iones y/o átomos de $\mathrm{Fe}$ y de $\mathrm{O}_{2}$ a través de la masa sólida, lo cual constituye una difusión en el estado sólido.

Los iones o átomos de $\mathrm{Fe}$ se difunden rápidamente a través de la red cristalina del oxigeno.

La difusividad disminuye con el aumento del oxígeno, lo cual produce una estructura más compacta.

6.5. Reacción química entre el $\mathrm{CO}$ y el sólido.

El mecanismo de la reducción de una red cristalina de hemática a $1000^{\circ} \mathrm{C}$ es la siguiente:

a) Ruptura de la red cristalina del FeO y formación de la fase metálica de $\mathrm{Fe}$ en la interfase $\mathrm{FeO}-\mathrm{Fe}$ $o$ en una interfase $\mathrm{FeO}-\mathrm{Fe}-\mathrm{CO}$, si la fase de hierro metálico fuera lo suficientemente porosa.

b) Difusión en el estado sólido de los iones de Fe o de átomos de $\mathrm{Fe}$ a través de la fase wustita $\mathrm{FeO}$ hacia la fase magnetita.

c) Difusión en el estado sólido de los iones o átomos de $\mathrm{Fe}$ dentro de la fase magnetita $\mathrm{Fe}_{3} \mathrm{O}_{4}$ sin alteración significativa de la red cristalina del oxido $\mathrm{Fe}_{3} \mathrm{O}_{4}$, de modo que hay una gradual conversión del $\mathrm{Fe}_{3} \mathrm{O}_{4}$ a $\mathrm{FeO}$.

d) Difusión en el estado sólido de los iones o átomos de $\mathrm{Fe}$ a través de la fase $\mathrm{Fe}_{3} \mathrm{O}_{4}$ 
e) Reacción $\mathrm{Fe}_{2} \mathrm{O}_{3} \quad \mathrm{Fe}_{3} \mathrm{O}_{4}$ en el limite de fase, produciéndose el cambio de la red hexagonal a cúbica compacta.

\section{DETERMINACIÓN DE LOS PARÁMETROS BÁSICOS DE DISEÑO}

Capacidad de tratamiento del Horno de Reacción Directa.

\begin{tabular}{|c|c|c|c|c|c|}
\hline $\begin{array}{l}\text { PESO DE MINERAL } \\
\text { (densidad verdadera } 2,5 \text { ) }\end{array}$ & $\mathrm{Kg}$ & 5 & 10 & 20 & 40 \\
\hline $\begin{array}{l}\text { Densidad aparente } \\
\text { mineral (malla }-4+6)\end{array}$ & $\begin{array}{c}\mathrm{Kg} / \mathrm{d}- \\
\mathrm{m} 3\end{array}$ & 1,5 & 1,5 & 1,5 & 1,5 \\
\hline Volumen de carga & $\mathrm{dm} 3$ & 3,3 & 6,6 & 13,3 & 26,6 \\
\hline $\begin{array}{l}\text { Longitud del horno (1 } \\
m-2 m)\end{array}$ & $\mathrm{dm}$ & 10 & 10 & 15 & 20 \\
\hline $\begin{array}{l}\text { Área del siguiente } \\
\text { circular }\end{array}$ & $\mathrm{dm} 2$ & 0,33 & 0,66 & 0,88 & 1,33 \\
\hline $\begin{array}{l}\text { Porcentaje del área } \\
\text { Total }\end{array}$ & $\%$ & 6 & 7 & 8 & 9 \\
\hline $\begin{array}{l}\text { Área de la sección } \\
\text { circular }\end{array}$ & $\mathrm{dm} 2$ & 5,5 & 9,4 & 11 & 14,8 \\
\hline \multirow[t]{2}{*}{$\begin{array}{l}\text { Diámetro interior } \\
\text { sección circular }\end{array}$} & $\mathrm{dm}$ & 2,6 & 3,5 & 3,7 & 4,3 \\
\hline & $\mathrm{cm}$ & 26 & 35 & 37 & 43 \\
\hline Espesor refractario & $\mathrm{cm}$ & $15 \times 2$ & $15 \times 2$ & $15 \times 2$ & $15 \times 2$ \\
\hline $\begin{array}{l}\text { Diámetro exterior } \\
\text { sección circular }\end{array}$ & $\mathrm{cm}$ & 56 & 65 & 67 & 73 \\
\hline \multirow[t]{2}{*}{ Volumen del horno } & $\mathrm{cm} 3$ & 24610 & 33166 & 52858 & 83665 \\
\hline & m3 & 2,4 & 3,3 & 5,3 & 8,3 \\
\hline \multicolumn{6}{|c|}{$\begin{array}{l}\text { OTROS FACTORES QUE DETERMINAN LA CAPACIDAD DE } \\
\text { TRATAMIENTO }\end{array}$} \\
\hline $\begin{array}{l}\text { Consumo de } \\
\text { combustible }\end{array}$ & Gal. & 10 & 12 & 18 & 27 \\
\hline $\begin{array}{l}\text { Rendimiento del } \\
\text { Quemador } \\
\end{array}$ & GPH & 4 & 4 & 7 & 7 \\
\hline $\begin{array}{l}\text { Diámetro Aire } \\
\text { Quemador }\end{array}$ & Pulga. & 1,5 & 1,5 & 2 & 2 \\
\hline Volumen de Gases & $\mathrm{m} 3 / \mathrm{h}$ & 140 & 160 & 250 & 380 \\
\hline Pérdida de Calor & $\mathrm{Kca} / \mathrm{h}$ & 250.000 & 300.000 & 460.00 & 700.000 \\
\hline $\begin{array}{l}\text { Seguridad contra } \\
\text { explosión }\end{array}$ & & ALTA & ALTA & MEDIA & MENOR \\
\hline Potencia del Motor & HP & 3 & 5 & 5 & 10 \\
\hline $\begin{array}{l}\text { Costo por Prueba } \\
\text { Unitaria }\end{array}$ & $\mathrm{S} /$. & 55 & 70 & 100 & 170 \\
\hline $\begin{array}{l}\text { Costo por Prueba ( } 3 \\
\text { Pruebas) }\end{array}$ & SI. & 47 & 60 & 85 & 145 \\
\hline
\end{tabular}

\subsection{FUNCIONAMIENTO}

La carga del horno está constituida por:

$\begin{array}{ll}\text { Mineral de hierro } & 5 \mathrm{Kg} \\ \text { Carbón } & 2,5 \mathrm{Kg} \\ \text { Caliza } & 0,7 \mathrm{Kg}\end{array}$

Lo cual permitirá obtener $3,6 \mathrm{~kg}$. de hierro esponja.

El mineral ingresa por la canaleta de alimentación a una granulometría de malla +10 y $-4(1,7-4,8$ $\mathrm{mm}$ ) a la zona de prerreducción, en donde a una temperatura de $700^{\circ} \mathrm{C}$, se desarrollan las siguientes reacciones:

$$
\begin{aligned}
& 3 \mathrm{Fe}_{2} \mathrm{O}_{3}+\mathrm{CO}=2 \mathrm{Fe}_{3} \mathrm{O} 4+\mathrm{CO}_{2}+15 \mathrm{Kcal} \\
& \mathrm{Fe} 3 \mathrm{O}_{4}+\mathrm{CO}=3 \mathrm{FeO}+\mathrm{CO}_{2}-11 \mathrm{Kcal}
\end{aligned}
$$

Logrando que las particulas de mineral estén constituidas por capas, siendo la externa de wustita $(\mathrm{FeO})$ que es porosa, a continuación magnetita $\left(\mathrm{Fe}_{3}\right.$ $\left.\mathrm{O}_{4}\right)$ y al centro la original hematita $\left(\mathrm{Fe}_{2} \mathrm{O}_{3}\right)$

Después de 3 horas de retención, se abre la compuerta de descarga y el mineral ingresa en el horno giratorio, en donde a una temperatura de 1000 ${ }^{\circ} \mathrm{C}$, se desarrollan las siguientes reacciones:

$$
\begin{array}{ll}
\mathrm{FeO}+\mathrm{CO}=\mathrm{Fe}+\mathrm{CO}_{2} & +3,2 \mathrm{Kcal} \\
\mathrm{CO}_{2}+\mathrm{C}=2 \mathrm{CO} & -41 \mathrm{Kcal}
\end{array}
$$

$\mathrm{FeO}+\mathrm{C}=\mathrm{Fe}+\mathrm{CO}-38 \quad \mathrm{Kcal}$

El gas reductor $\mathrm{CO}$ actúa sobre el $\mathrm{FeO}$, produciéndose hierro metálico y $\mathrm{CO}_{2}$.

El $\mathrm{CO}_{2}$ se combina con el carbón formando 2 moles de $\mathrm{CO}$, que vuelven a actuar sobre el $\mathrm{FeO}$. Se gira el horno a 2RPM.

El resultado es la reacción (5), que se dencmina REACCIÓN DE REDUCCIÓN DIRECTA, pero que en realidad es la suma teórica de dos reacciones reales: la (3) y la (4), en la que la reacción (4) es la regeneración del $\mathrm{CO}$, que es el que realiza la reducción de la wustita $(\mathrm{FeO})$ y que produce la metalización de la partícula de mineral. 
Después de 3 horas, la metalización alcanza un valor de $85 \%$, retirándose el hierro esponja producido por la compuerta de salida del horno a través de un ducto que descarga en un recipiente cerrado y refrigerado por agua para enfriar el hierro esponja hasta temperatura ambiente.

\subsection{BALANCE DE CARGA Y ENERGÍA}

\section{a. Balance de Carga (Kg.)}

\begin{tabular}{|l|c|l|c|}
\hline \multicolumn{2}{|c|}{ ENTRADA } & \multicolumn{2}{c|}{ SALIDA } \\
\hline Mineral & 5 & Hierro Esponja & 3,60 \\
\hline Fundente & 0,7 & Carbón & 1,88 \\
\hline Carbón & 2,5 & Gases & 473,72 \\
\hline Petróleo & 30,0 & & \\
\hline Aire & 441,0 & & \\
\hline Total & 479,2 & & 479,20 \\
\hline
\end{tabular}

b. Balance de Energía (Kcal)

\begin{tabular}{|c|c|c|c|}
\hline \multicolumn{2}{|c|}{ ENTRADA } & \multicolumn{2}{|l|}{ SALIDA } \\
\hline $\begin{array}{l}\text { CALOR } \\
\text { APORTADO } \\
\end{array}$ & 379520 & $\begin{array}{l}\text { Calor de reducción } \\
\mathrm{Fe} 2 \mathrm{O}^{3}+3 \mathrm{CO}=2 \mathrm{Fe}+3 \mathrm{CO}_{2}\end{array}$ & 3000 \\
\hline & & $\begin{array}{l}\text { Calor de formación } \\
\mathrm{CO}_{2}+\mathrm{C}=2 \mathrm{CO}\end{array}$ & 137320 \\
\hline & & $\mathrm{CO}_{2}+\mathrm{C}=2 \mathrm{CO}$ & 23760 \\
\hline & & $\begin{array}{l}\text { Calor sensible del Hierro } \\
\text { Esponja }\end{array}$ & 5600 \\
\hline & & $\begin{array}{l}\text { Calor sensible de la } \\
\text { carcaza }\end{array}$ & 1440 \\
\hline & & $\begin{array}{l}\text { Perdida por paredes del } \\
\text { horno }\end{array}$ & 56592 \\
\hline & & Perdida en los gases & 151808 \\
\hline TOTAL & 379520 & TOTAL & 379520 \\
\hline
\end{tabular}

\section{COSTO DE CONSTRUCCIÓN}

\begin{tabular}{|l|l|c|c|c|c|}
\hline \multicolumn{1}{|c|}{ ELEMENTO } & MATERIAL & UNID. & CANT. & PREC SI & TOTALSI. \\
\hline Soporte del homo & Angulo $11^{\prime \prime} \times 1 / 8^{\prime \prime}$ & $c / u$ & 6,75 & 20 & 135 \\
\hline Soporte quemador & Angulo $1 " \times 1 / 8^{\prime \prime}$ & $c / u$ & 2,60 & 12 & 30 \\
\hline Carcaza horno & Plancha met 1/8" & $c / 4$ & 1,00 & 175 & 175 \\
\hline Sist aliment y enfria & Plancha met $1 / 8^{\prime \prime}$ & $c / u$ & 1,00 & 175 & 175 \\
\hline Sist ingreso aire & Tubo 2" & $c / u$ & 0,20 & 100 & 20 \\
\hline & Tubo 1" & $c / 4$ & 0,40 & 40 & 16 \\
\hline Rodillos & & $c / u$ & 4 & 30 & 120 \\
\hline Quemador & & $c / u$ & 1 & 300 & 300 \\
\hline Compuerta aliment & & $c / u$ & 1 & 30 & 30 \\
\hline Motor de 3 HP & & $c / u$ & 1 & 300 & 300 \\
\hline Ventilador & & $c / u$ & 1 & 500 & 500 \\
\hline Refractario & Arena Refractaria & bolsas & & 200 & 200 \\
\hline Energia & & & & & 60 \\
\hline Pintado & & & & & 60 \\
\hline Soldadura & Electrodos & $\mathrm{Kg}$. & 10 & 6 & 60 \\
\hline Rolado & & & & & 100 \\
\hline Tanque Alim. Petr. & & & & & 150 \\
\hline Mano de obra & & & & & 500 \\
\hline TotAL & & & & & 3231 \\
\hline
\end{tabular}

10.DISCUSIÓN Y ANÁLISIS DE RESULTADOS

- El equipo que se ha diseñado permitirá efectuar pruebas con los diferentes minerales de hierro de la zona, dado que será posible ajustar dentro de un amplio rango las variables de operación.

- Una de las principales variables a investigarse es la granulometría del mineral, permitiendo el equipo hacerlo hasta un tamaño de $1.7 \mathrm{~mm}$ (malla 10) como mínimo y un máximo de $1 / 2$ pulgada, sólo que el tiempo de retención dentro del horno se extendería a más de 6 horas.

- El equipo se considera seguro contra explosiones, dado que será operado por los alumnos bajo la supervisión del profesor especialista.

\section{CONCLUSIONES}

1. El Horno Piloto de reducción directa permitirá realizar prácticas académicas dentro del sistema universitario, relacionadas con la producción de Hierro Esponja.

2. Se podrán realizar trabajos de investigación en los siguientes campos:

a) Empleo del Hierro Esponja en la cementación del cobre a partir de soluciones de sulfato de cobre. 
b) Reducción del costo de producción del Hierro Esponja por debajo del precio de la chatarra, mediante la reducción de la granulometria y diversificación de procesos, para que se constituya en una alternativa de la chatarra.

3. Se podrán realizar pruebas con los diferentes minerales de hierro de la zona de Tacna y de las regiones aledañas, con la finalidad de elaborar estudios técnicos y promover su explotación.

4. El rendimiento del horno es bajo, por la pequeña cantidad de la muestra, pero permite estudiar las variables del proceso.

5. Es posible adicionar un sistema de recuperación de calor para mejorar el rendimiento térmico del horno y reducir el costo de las pruebas.

6. Las pruebas efectuadas en este equipo permitirán realizar el escalamiento para determinar el costo de producción y la construcción de equipos de mayor capacidad.

7. El grado de metalización estará en función de la pureza del mineral de hierro, sobre todo en cuanto a contenido de sílice, alúmina y azufre; comparando la calidad del mineral con laq calidad de un pellets se puede apreciar la diferencia que habría en el producto.

\begin{tabular}{|l|c|c|c|c|c|c|c|c|c|}
\hline & $\mathrm{Fe}$ & $\mathrm{SiO}_{2}$ & $\mathrm{Al}_{2} \mathrm{O}_{3}$ & $\mathrm{CaO}$ & $\mathrm{MgO}$ & $\mathrm{P}$ & $\mathrm{S}$ & $\mathrm{Mn}$ & 0 \\
\hline Hematita & 62 & 5 & 1 & 5 & & & & 0.5 & 26 \\
\hline Pellets & 67.68 & 0.7 & 0.5 & 0.18 & 0.56 & 0.01 & 0.05 & 0.14 & 30 \\
\hline
\end{tabular}

\section{REFERENCIAS BIBLIOGRÁFICAS}

H.M.Boylston. : Introducción a la Metalurgia del Hierro y del Acero. Edit. Wiley, USA. 1990.

José Apraiz Barreiro.: Fabricación del Hierro Acero y Fundiciones. 1990, Edit. Urmo S.A., España.

J. S. Kirkaldy and R.G.Ward. : Aspects of Modern Ferrous Metallurgy. Edición 1984, Blackie and son Limited.

H. Appold, K. Feiler.: Tecnologia de los Metales. Edit Reverté, España, 1994. 